\section{EFFECTIVENESS AND UTILIZATION OF FIRST-LINE IMMUNE CHECKPOINT INHIBITORS FOR PATIENTS WITH EXTRACRANIAL \& INTRACRANIAL METASTATIC MELANOMA}

${ }^{1}$ Nayan Lamba, ${ }^{2}$ Bryan lorgulescu*. 'Harvard Medical School, Boston, MA, USA; ${ }^{2}$ DanaFarber Cancer Institute, Boston, MA, USA

Background We previously demonstrated the effectiveness of 2nd-line immune checkpoint inhibitors (ICI) for stage 4 melanoma patients. ${ }^{1}$ In late 2015, ICI was FDA-approved and NCCN-recommended in the 1 st-line setting. ${ }^{3}{ }^{4}$ Here we assess the real-world effectiveness and utilization of 1 st-line ICI among advanced melanoma patients following 2015.

Methods Patients newly-diagnosed with stage 4 melanoma during 2010-2018 were identified using the U.S. National Cancer Database (comprises $>70 \%$ of newly-diagnosed cancers). ${ }^{5}$ Postapproval 1st-line ICI's overall survival (OS, estimated by Kaplan-Meier techniques) and utilization were assessed for patients diagnosed in 2016-2018, using multivariable Cox and logistic regression, respectively. To account for immortal time bias in receiving ICI, we only included those patients in regression analyses who survived at least until the landmark timepoint, defined as the median time from diagnosis to ICI initiation (49 days). ${ }^{1}{ }^{2}$ The more conservative 75 th percentile diagnosis-to-ICI-initiation landmark timepoint (80 days) was also evaluated. Analyses were adjusted for patient, tumor, treatment, socioeconomic, and care setting characteristics.

Results Among 14,912 stage 4 melanoma patients, 1st-line immunotherapy utilization increased from $8.4 \%$ in 2010 to $39.2 \%$ in 2015 , and $57.9 \%$ in 2018.(Figure 1) Altogether, median OS improved from $8.0 \operatorname{mos}(95 \% \mathrm{CI}=7.3-8.8)$ in 2010 to $16.1 \mathrm{mos}(95 \% \mathrm{CI}=14.0-18.5)$ in 2017 . For patients diagnosed in 2016+ who survived at least until the landmark timepoints, OS improved with 1st-line ICI (median OS $=33.1$ mos, $95 \% \mathrm{CI}=29.4-40.5$; vs just $13.6 \operatorname{mos}$ for no $\mathrm{ICI}, 95 \%$ $\mathrm{CI}=12.1-16.1 ; \quad \mathrm{HR}=0.58, \quad 99 \% \mathrm{CI}=0.50-0.68 ; \quad \operatorname{padj}<0.001)$ (figure 2A-B) - even after adjusting for patient, disease, and treatment factors, and using either landmark.(Table 1) This included patients with either brain metastases (ICI median $\mathrm{OS}=16.7 \mathrm{mos}, 95 \% \mathrm{CI}=13.1-19.9$; vs no $\mathrm{ICI}=7.8 \mathrm{mos}, 95 \%$ $\mathrm{CI}=6.8-9.0$; padj $<0.001$ ) or lung metastases (ICI median $\mathrm{OS}=26.0 \mathrm{mos}, 95 \% \mathrm{CI}=20.0-33.0 ;$ vs no $\mathrm{ICI}=9.3 \mathrm{mos}, 95 \%$ $\mathrm{CI}=8.0-10.5$; padj<0.001). (Figure 2C-F)We then used multivariable logistic regression (with landmark timepoints to reduce bias from early mortality) to identify putative barriers to receiving 1st-line ICI in 2016+. Advanced melanoma patients who had more comorbidities or brain metastases, or who were older, uninsured/Medicaid-insured, from the poorest quartile of households, or managed at community hospitals were less likely to receive ICI.(all $\mathrm{p}<0.05$, table 2)

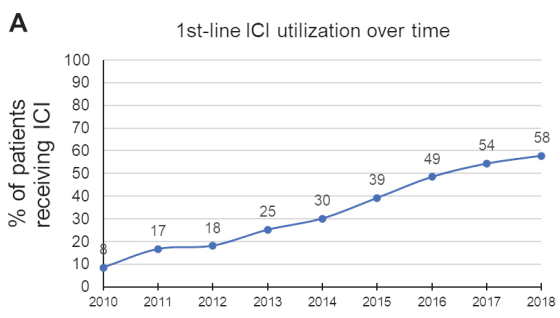

B 1st-line ICl utilization over time, for Brain \& Lung mets
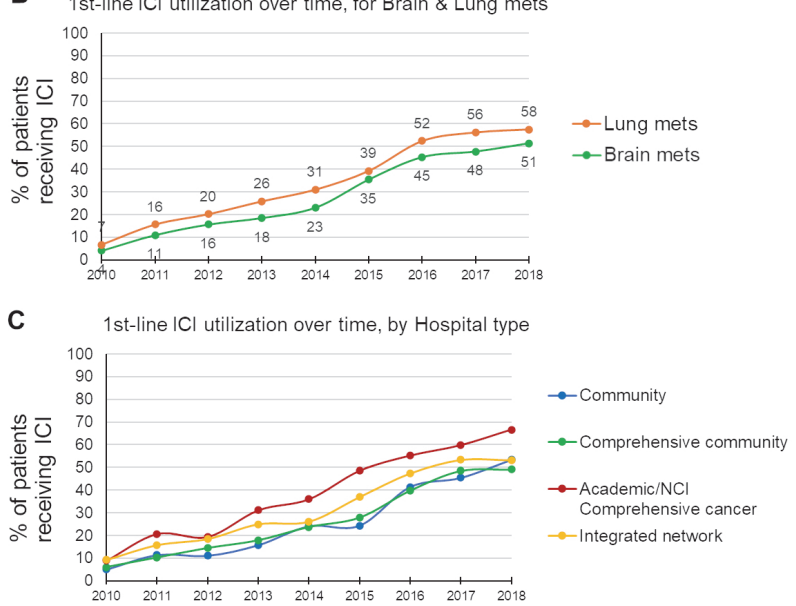

Abstract 246 Figure 1 (A) The percent of stage 4 melanoma patients diagnosed each year who received 1st-line immune checkpoint inhibitor (ICI), with stratification by (B) brain or lung metastatic involvement, and (C) treating hospital type.

Abstract 246 Table 1 Multivariable Cox regression analysis of overall survival among stage 4 melanoma patients in 2016+. To account for immortal time bias, landmark timepoints were utilized, defined by the median (i.e. 49d; panels $A, C, E$ ) and 75th percentile (i.e. 80d; panels $\mathrm{B}, \mathrm{D}, \mathrm{F}$ ) time from diagnosis to $\mathrm{ICl}$ initiation. Patients had to survive at least as long as the landmark timepoint to be included in the analysis. Results are shown for all stage 4 patients, as well as those with brain or lung metastases. $p$ values are only displayed for the primary association of interest.

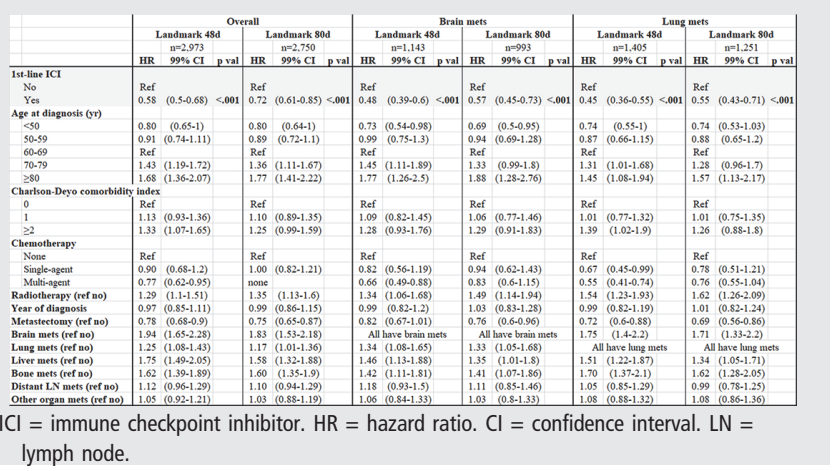


Abstract 246 Table 2 Multivariable logistic regression analysis of 1 st-line immune checkpoint inhibitor (ICI) receipt among stage 4 melanoma patients in 2016+. To account for bias due to early mortality, landmark timepoints were utilized, defined by the median (i.e. 49d) and 75th percentile (i.e. 80d) time from diagnosis to $\mathrm{ICl}$ initiation. Patients had to survive at least as long as the landmark timepoint to be included in the analysis. Results are shown for all stage 4 patients, as well as those with brain or lung metastases. Variables that demonstrate a significant association with $\mathrm{ICl}$ receipt are highlighted in yellow.

\begin{tabular}{|c|c|c|c|c|c|c|}
\hline & \multirow{2}{*}{\multicolumn{3}{|c|}{ Landmark 48d }} & \multirow{2}{*}{\multicolumn{3}{|c|}{ Landmark 80d }} \\
\hline & & & & & & \\
\hline & OR & $99 \%$ CI & p val & OR & $99 \% \mathrm{CI}$ & p val \\
\hline \multicolumn{7}{|l|}{ Age at diagnosis (yr) } \\
\hline $\begin{array}{l}<50 \\
50-5\end{array}$ & 1.33 & $\begin{array}{l}(0.91-1.94) \\
(0.74-1.33)\end{array}$ & $\begin{array}{c}0.05 \\
0.958\end{array}$ & $\begin{array}{l}1.34 \\
0.99\end{array}$ & $\begin{array}{l}(0.91-1.97) \\
(0.74-134)\end{array}$ & $\begin{array}{l}0.06 \\
0.96\end{array}$ \\
\hline $\begin{array}{l}50-59 \\
60-69\end{array}$ & Ref & & & Ref & & \\
\hline $70-79$ & 0.78 & $(0.59-1.03)$ & 0.02 & 0.84 & $(0.63-1.13)$ & 0.13 \\
\hline$\geq 80$ & 0.44 & $(0.32-0.61)$ & $<.001$ & 0.45 & $(0.32-0.63)$ & $<.001$ \\
\hline \multicolumn{7}{|l|}{ Sex } \\
\hline Male & Ref & & & Ref & & \\
\hline Female & 0.91 & $(0.74-1.12)$ & 0.253 & 0.90 & $(0.72-1.11)$ & 0.19 \\
\hline \multicolumn{7}{|l|}{ Race/Ethnicity } \\
\hline White, non-Hispanic & Ref & & & Ref & & \\
\hline Black, 1 & 1.45 & $(0.65-3.26)$ & 0.235 & 1.47 & $(0.64-3.37)$ & 0.23 \\
\hline Asian & 93 & $(0.29-2.96)$ & 0.874 & 0.88 & $(0.27-2.82)$ & 0.77 \\
\hline Hispani & 0.78 & $(0.43-1.43)$ & 0.292 & 0.77 & $(0.41-1.46)$ & 0.3 \\
\hline Other & 29 & $(0.47-3.56)$ & 0.51 & 1.27 & $(0.41-3.89)$ & 0.58 \\
\hline $\mathrm{n} / \mathrm{a}$ & 0.77 & $(0.2-2.99)$ & 0.616 & 0.72 & $(0.18$ & 0.54 \\
\hline \multicolumn{7}{|c|}{ Charlson-Deyo comorbidity index } \\
\hline & Ref & & & Ref & & \\
\hline 1 & 0.91 & $(0.69-1.19)$ & 0.356 & 0.91 & $(0.6$ & 0.42 \\
\hline$\geq 2$ & 0.72 & $(0.53-0.98)$ & 0.007 & 0.75 & $(0.54-$ & 0.03 \\
\hline tear of $\mathrm{c}$ & 1.10 & $(0.98-1.24)$ & 0.039 & 1.02 & $(0.9-1.15)$ & 0.72 \\
\hline \multicolumn{7}{|l|}{ Primary skin site } \\
\hline Lip & 71 & $(0.05-10.6)$ & 0.741 & 0.63 & $(0.04-9.61)$ & 0.66 \\
\hline Eyelic & 0 & $(0.02-3)-10$ & 0.074 & 0.18 & $(0.02$ & 0.06 \\
\hline Ext & & $(0.5$ & 0.235 & 2.29 & & 0.11 \\
\hline Face & 9 & $(0.47-1.69)$ & 0.651 & 0.85 & $(0.44$ & 0.54 \\
\hline Scal & 16 & $(0.73-1.85)$ & 0.406 & 1.18 & $(0.73-1.91)$ & 0.38 \\
\hline Tru & ef & & & Ref & & \\
\hline Uppe & 6 & $(0.62-1.49)$ & 0.813 & 0.92 & & 0.62 \\
\hline Low & 3 & $(0.97$ & 0.017 & 1.56 & $(0.9$ & 0.02 \\
\hline & & & 0.329 & 3.04 & & 0.18 \\
\hline Skin, I & 1 & $(0.64$ & 0.498 & 0.88 & $(0.6$ & 0.37 \\
\hline ef no) & 3 & $(0.81-$ & 0.777 & 1.08 & 4) & 0.44 \\
\hline & & & $<.001$ & 1.59 & & $<.001$ \\
\hline Bone $\mathrm{m}$ & 55 & $(0.8$ & 0.584 & 1.12 & $(0.8$ & 0.25 \\
\hline rain $\mathbf{m}$ & 55 & $(0.43-0.7)$ & $<.001$ & 0.57 & $(0.44-0.74)$ & $<.001$ \\
\hline & & & $<.001$ & 1.59 & & $<.001$ \\
\hline Other o & 0.99 & $(0.81-1.22)$ & 0.945 & 0.98 & $(0.8-1.21)$ & 0.82 \\
\hline Primary si & & & & & & \\
\hline & 6 & & 0.733 & 1.00 & & 0.99 \\
\hline Exci & 1.02 & $(0.7-1.48)$ & 0.884 & 1.07 & $(0.72-1.57)$ & 0.67 \\
\hline Gr & Ref & & & Ref & & \\
\hline adio & 1.91 & $(1.5-2$. & $<.001$ & 1.92 & $(1.49-2.49)$ & $<.001$ \\
\hline & & & & & & \\
\hline None & Ref & & & Ref & & \\
\hline & & & $<.001$ & 0.18 & & $<$ \\
\hline & & & $<.001$ & 0.10 & & $<.001$ \\
\hline Ietast & 1.03 & $(0.81$ & 0.731 & 1.06 & $(0.83-1.36)$ & 0.5 \\
\hline & & & & & & \\
\hline No & 0.60 & $(0.36$ & 0.01 & 0.63 & $(0.37-1.09)$ & 0.03 \\
\hline & Ref & & & Ref & & \\
\hline & & & $<$ & 0.56 & & \\
\hline & & & 0.02 & 0.79 & & 0.03 \\
\hline Otl & & $(0.18$ & $<.001$ & 0.35 & $(0.1$ & $<.001$ \\
\hline Unkno & 0.62 & $(0.24$ & 0.194 & 0.70 & $(0.24-1.99)$ & 0.38 \\
\hline Mediar & of ati & 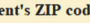 & & & & \\
\hline & Ref & & & Ref & & \\
\hline & & & & 1.36 & & 0.02 \\
\hline & & $(0.84$ & 0.26 & 1.15 & $(0.8$ & 0.27 \\
\hline$y=$ & 1.40 & $(1.03-1.9)$ & 0.005 & 1.52 & $(1.1-2.09)$ & $<.001$ \\
\hline Iospi & & & & & & \\
\hline Con & 5 & & 0.007 & 0.68 & & 0.0 \\
\hline & 0.58 & $(0.46-0.72)$ & $<.001$ & 0.57 & $(0.45-0.72)$ & $<$ \\
\hline & Ref & & & Ref & & \\
\hline & 0.70 & $(0.53-0.92)$ & .00 & 0.69 & (0. & \\
\hline & & & & & & \\
\hline & 1.46 & & 0.058 & 1.47 & & 0.0 \\
\hline & 1.20 & $(0.85-1$. & 0.169 & 1.25 & $(0.86-1.79)$ & 0.12 \\
\hline & \begin{tabular}{|l|l|}
1.07 \\
\end{tabular} & $(0.79-1.46)$ & 0.56 & 1.09 & $(0.79-1.51)$ & 0.47 \\
\hline & Ref & & & ef & & \\
\hline & 1.08 & $(0$. & 0.63 & 1.18 & & 0.3 \\
\hline & 1.59 & & & 1.71 & & \\
\hline u & 1.20 & & 0.242 & 1.19 & & 0.29 \\
\hline & & & 0.024 & 1.58 & 57) & 0.02 \\
\hline & & & 0.255 & 1.1 & & 0.36 \\
\hline
\end{tabular}

$\mathrm{ICI}=$ immune checkpoint inhibitor. $\mathrm{OR}=$ odds ratio. $\mathrm{Cl}=$ confidence interval. $\mathrm{LN}=$ lymph node.
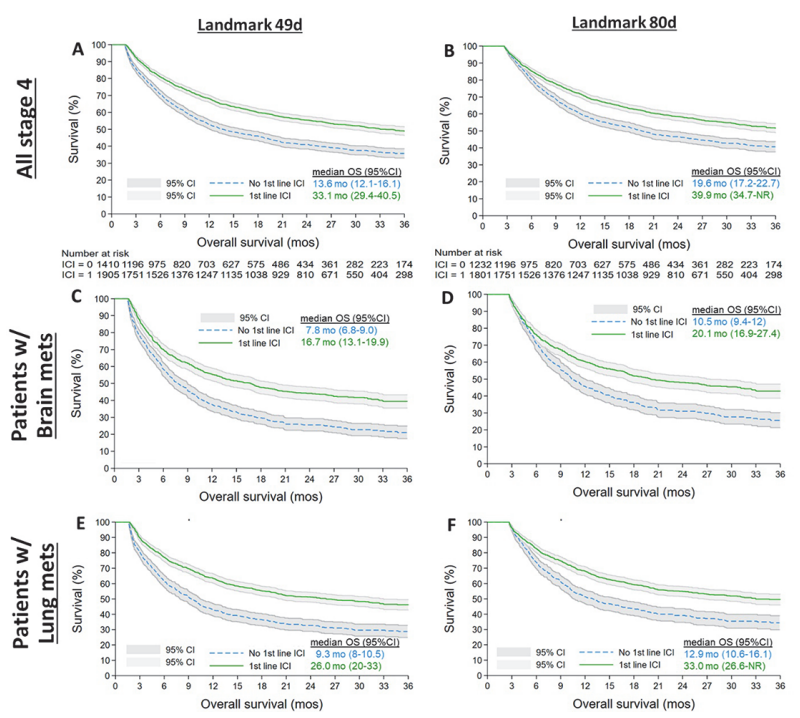

Abstract 246 Figure 2 Overall survival associated with 1st-line immune checkpoint inhibitors (ICI) for stage 4 melanoma patients diagnosed after 2015 (A-B), including patients with brain (C-D) and lung metastases (E-F). To account for immortal time bias, landmark timepoints were utilized, defined by the median (i.e. 49d; panels $A, C$, E) and 75th percentile (i.e. 80 d; panels $B, D, F$ ) time from diagnosis to $\mathrm{ICl}$ initiation. Patients had to survive at least as long as the landmark timepoint to be included in the analysis.

Conclusions Following FDA-approval in 2015, 1st-line ICI was associated with dramatic improvements in OS for stage 4 melanoma patients-including those with brain or lung metastases. As of 2018, 42\% of patients still weren't receiving 1st-line ICI in the U.S.- particularly patients who were underinsured, from the poorest quartile of households, or managed at community hospitals - suggesting that disparities exist in guidelinerecommended 1st-line ICI utilization for advanced melanoma patients.

\section{REFERENCES}

1.. Dobry A, Zogg C, Hodi F, Smith $T$, Ott P, lorgulescu JB. Management of metastatic melanoma: improved survival in a national cohort following the approvals of checkpoint blockade immunotherapies and targeted therapies. Cancer Immunol Immunother 2018 December;67(12):1833-1844.

2.. Iorgulescu JB, Harary M, Zogg C, Ligon K, Reardon D, Hodi F, Aizer A, Smith T. Improved risk-adjusted survival for melanoma brain metastases in the era of checkpoint blockade immunotherapies: results from a national cohort. Cancer Immunol Res 2018 September;6(9):1039-1045.

3.. Beaver JA, Theoret MR, Mushti S, He K, Libeg M, Goldberg K, Sridhara R, McKee AE, Keegan P, Pazdur R. FDA approval of nivolumab for the first-line treatment of patients with BRAFV600 wild-type unresectable or metastatic melanoma. Clin Cancer Res 2017 July 15;23(14):3479-3483

4.. National Comprehensive Cancer Network Clinical Practice Guidelines in Oncology. Melanoma, Version 1.2016.

5.. Boffa DJ, Rosen JE, Mallin K, Loomis A, Gay G, Palis B, Thoburn K, Gress D, McKellar DP, Shulman LN, Facktor MA, Winchester DP. Using the national cancer database for outcomes research: a review. JAMA Oncol 2017 December 1:3 (12):1722-1728.

http://dx.doi.org/10.1136/jitc-2021-SITC2021.246 